\title{
Foveomacular retinitis
}

\author{
B S KUMING \\ From Johannesburg, South Africa
}

SUMmARY A group of patients is described who developed the clinical features of foveomacular retinitis. No causative factors were isolated, and all patients strongly denied any type of sun gazing. It is possible that there is a group of patients who have the features of foveomacular retinitis but have not had any direct exposure to the sun. These patients would then constitute a primary type of foveomacular retinitis, as opposed to a secondary type which has a known cause and is synonymous with solar retinopathy.

Foveomacular retinitis (FMR) ${ }^{12}$ is a disease involving the posterior pole of the eye in which characteristic foveal lesions develop. The term has been used synonymously with solar retinopathy, ${ }^{2,-5}$ a condition with a specific fundal appearance and associated with a characteristic history of direct gazing at the sun or observation of an eclipse. However, it has also been described in patients who have denied sun gazing. ${ }^{6}$

\section{Cases}

This paper describes a series of 10 patients who, while showing the features of foveomacular retinitis, did not have any history of exposure to an eclipse, direct sun gazing, or trauma. The aetiology remains unknown, and the condition does not fit into any of the categories of subtle macular changes similar in appearance to foveomacular retinitis.

The patients' ages ranged from 16 to 57 . There were six females and four males. Three cases were bilateral (1 F, $2 \mathrm{M})$ and seven were unilateral (4 RE, $3 \mathrm{LE}$ ). The vision varied between $20 / 25$ and $20 / 30$. The length of history varied from a few months in the younger patients to many years in the older patients. There was no history of trauma, sun gazing, or eclipse observation. No precipitating factors were noted, and none of the patients could recollect any coexisting viral infection at the time of onset.

A uniform symptom was an inability to see the 'whole'. Letters jumped or were missing from the area being observed. The actual slight drop in vision did not disturb the patients. These findings were confirmed on Amsler grid charting, which showed

Correspondence to Dr B S Kuming, 716 Tower Hill, Cor/H/v Kotze \& Klein Strect, Hillbrow 2001, Johannesburg, South Africa small central defects in the affected eyes. The length of follow-up extended from six months to six years.

All patients were examined at least twice, with a minimum of six months between the two visits. In none, was there any alteration of the central macular area. All patients had biomicroscopy and contact lens examinations. Fluorescein angiography was performed on six patients. Two patients had a full battery of tests for uveitis. The results were negative. No peripheral fundal lesions were noted, and tonometry was normal in all patients.

The fundal area was typical, with minor variations (Fig. 1). At the macula a small red granular spot was

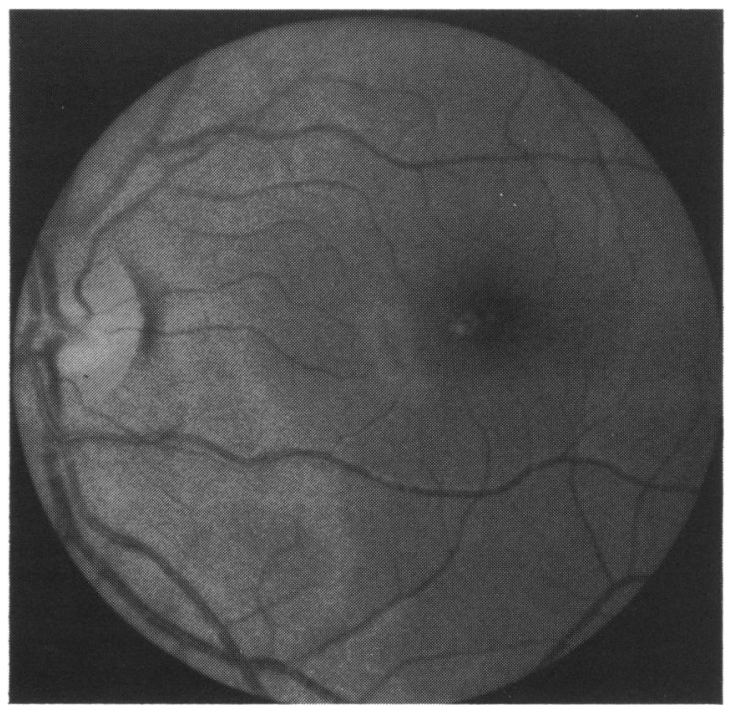

Fig. 1 Dark red foveal lesion. 


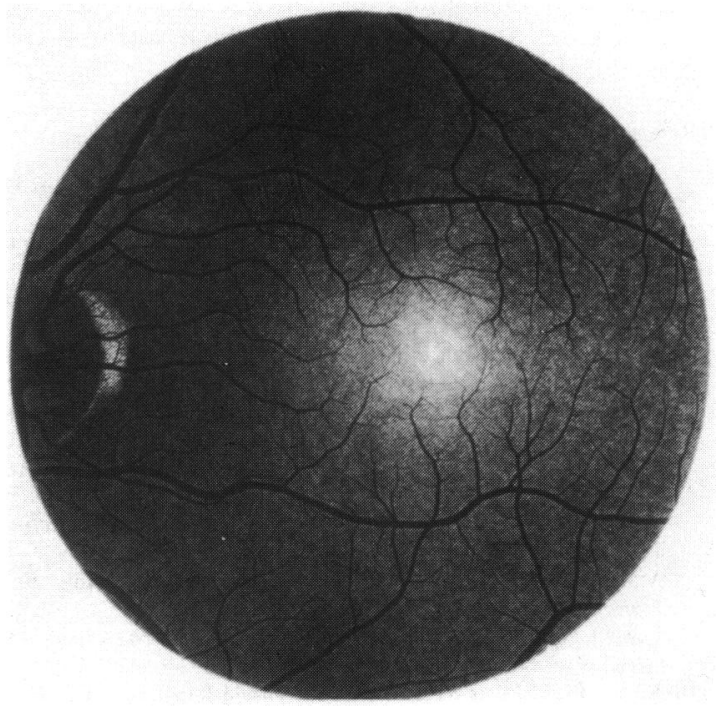

Fig. 2 Late stage angiogram showing faint ring shaped window defect.

noted. This might be linear or irregularly shaped. On superficial inspection it seemed to be a deep retinal haemorrhage. Closer examination showed that it was probably a small lamellar hole. It was, however, extremely difficult to be sure of the depth of the lesion. Surrounding this central area was a zone of disturbed macular pigment, with a rather mottled appearance. Irregular foveal reflexes were noted, and depending on the angle of the incident beam one or two yellowish reflexes or dots adjacent to the red spot were seen. The lesion appeared to change subtly as the incident beam of the slit-lamp was moved during contact lens examination. No evidence of serous detachment or vitreous cells were noted.

The fluorescein angiogram (Fig. 2) showed a ring of pigment epithelial damage appearing as a faint window defect. It was seen in every patient on whom an angiogram was done, and was thus unlike the lesions described in solar retinopathy, of which only severe cases show angiographic abnormalities. No full thickness foveal holes were noted.

\section{Discussions}

It is difficult to differentiate FMR from solar retinopathy, because the fundal appearance of both conditions are so similar. In solar retinopathy there is a history of recent viewing of an eclipse, or direct gazing at the sun by malingerers, sunbathers, nudists, drug addicts, and other cultists. A recent history of arc welding may also be given. Dhir et al. ${ }^{7}$ grouped the fundal lesions of solar retinopathy into three clinical subgroups: mild-dark brown granular lesion at the macula without macular oedema, situated perifoveally; moderate-reddish brown granular area surrounded by a grey ring of perifoveal retinal oedema; severe-greyish white patch due to severe oedema, and situated parafoveally. The fluorescein angiogram was usually normal, but a transmission defect or microleak in the parafoveal area occurred in some of the severe cases. Retinal oedema might mask the choroidal fluorescence, and the xanthophyllic pigment of the macular area might also mask minor pigment epithelial damage.

The severe burns usually resolved into a foveomacular hole with pigment clumping and mottling. In the late state two types of holes have been described, namely, a true lamellar hole due to necrosis of the inner retinal layers, and a pseudolamellar hole due to acute necrosis of pigment epithelial cells with formation of a pigment epithelial cyst. The appearance is that of a dark red or brownish hole-like lesion. It may also appear reddish-white with small yellow exudates in the centre of the lesion. ${ }^{3}$

The angiograms of the patients described in this series are identical to the angiograms of those patients described by Dhir et al. ${ }^{7}$ as having severe solar retinopathy. His patients had variable loss of vision and were described as belonging to his severe group; despite the severity of the lesions, most recovered to $6 / 6$ vision. The patients described here also had minimal loss of vision despite their angiographic abnormalities.

Most patients with solar retinopathy seem to recover fully or return to near normal acuity, though some cases do have a poorer prognosis. ${ }^{\text {}}$ Foveomacular retinitis was first described by Cordes ${ }^{1}$ in 1944, and then by Kerr and Little in $1966 .{ }^{2}$ All their cases were in young naval personnel, all of whom denied sun gazing or trauma. A yellowish foveal exudate developed which was surrounded by a grey zone of parafoveal oedema. After 14 days this disappeared, leaving a reddish foveal depression which became a foveal hole. The vision varied between 20/50 and 20/400, with no improvement in the cases with poor vision. Of great significance is the fact that some patients developed lesions in their second eye while confined to their ward in the hospital. Kerr and Little thought that the disease resulted from a localised choriocapillary vascular disease, with acute necrosis of the fovea.

Some authors ${ }^{34}$ consider that FMR and solar retinopathy are one and the same condition. However, some $42 \%$ of their cases did not have a history of sun exposure of any type. Woldoff and Kilpatrick ${ }^{6}$ also described three patients, all females, who strongly denied sun gazing and in whom FMR developed. 
Several other conditions may mimic foveomacular retinitis; they are rare. The appearance of the lesion and a careful history will distinguish these cases from those of solar retinopathy or FMR. They include ocular contusion" and whiplash maculopathy" due to tractional forces at the vitreoretinal interface, a congenital variation of macular anatomy, toxic maculopathy; acute retinal pigment epithelitis," 12 and acute macular neuroretinopathy. ${ }^{1314}$

In our patients the natural history was that of a sudden but minimal loss of vision. The visual loss did not vary and there was no recovery to normal. Patients adapted to this loss without difficulty. No family history was noted. All patients strongly denied exposure to an eclipse or sun gazing. The aetiology of the condition is unknown. Hereditary, known inflammatory, and traumaticinfluences have been excluded. It is possible that accidental exposure to excessive levels of the visible light spectrum could cause the lesions, but all patients denied such a possibility.

It would thus appear that the fundal appearances described in both FMR and solar retinopathy are identical. Two subgroups, however, seem to exist. In the first or primary type there are none of the aetiological features associated with solar retinopathy. The fluorescein angiogram shows mild abnormalities and the vision is usually around $20 / 30$. In the second group there is a definite history of exposure to excessive levels of the visible light spectrum.
Fluorescein angiographic abnormalities are usual in severe cases and, if present, may be associated with considerable loss of vision.

\section{References}

1 Cordes FC. A type of foveomacular retinitis observed in US Navy. Am J Ophthalmol 1944; 27: 803

2 Kerr TV, Little HL. Foveomacular retinitis. Arch Ophthalmol 1966; 76: 498.

3 Wegeland FL, Brenner EH. Solar retinopathy and foveomacular retinitis. Ann Ophthalmol 1975; 4: 445-503.

4 Ewald RA, Ritchey CL. Sungazing as the cause of foveomacular retinitis. Am J Ophthalmol 1970; 70: 491-7.

5 Gass JDM. Stereoscopic atlas of macular disease. St Louis: Mosby, 1977: 322-3.

6 Woldoff HS, Kilpatrick WRJ. Foveomacular retinitis. Ann Ophthalmol 1975; 7: 1035-8.

7 Dhir SP, Gupta A, Jain IS. Eclipse retinopathy. BrJ Ophthalmol 1981; 65: 42-5.

8 MacFaul PA. Visual prognosis after solar retinopathy. $\mathrm{Br} J$ Ophthalmol 1963; 53: 534-41.

9 Daily L. Macular and visual disturbances produced by traumatic vitreous rebound. South Med J 1971; 63: 1197-9.

10 Kelley JS, Hoover RE, George T. Whiplash maculopathy. Arch Ophthalmol 1976; 96: 834-5.

11 Krill AE, Deutman AR. Acute retinal epithelitis. Am J Ophthalmol 1972; 74: 193-205.

12 Friedman MW. Bilateral acute retinal pigment epithelium. Am J Ophthalmol 1975; 79: 567-70.

13 Boss PJM, Deutman AR. Acute macular neuro-retinopathy. Am J Ophthalmol 1975; 80: 573-84.

14 Rush JA. Acute macular neuro-retinopathy. Am J Ophthalmol 1977; 83: 490-4.

Accepted for publication 14 February 1986. 